\title{
Auricular Hillock
}

National Cancer Institute

\section{Source}

National Cancer Institute. Auricular Hillock. NCI Thesaurus. Code C34110.

Six small swellings on the embryonic visceral arch, which are derived from the first and second pharyngeal arches. The ear auricle develops from the fusion of these six auricular hillocks. 